\title{
Importancia de la diversidad de abejas (Hymenoptera: Apoidea) y amenazas que enfrenta en el ecosistema tropical de Yucatán, México \\ Importance of diversity of bees (Hymenoptera: Apoidea) and threats facing the tropical ecosystem of Yucatan, Mexico
}

\author{
Martínez-Puc Jesús Froylán ${ }^{1 *}$, Merlo-Maydana Flavio Eudaldo²
}

\begin{tabular}{|c|}
\hline Datos del Artículo \\
\hline $\begin{array}{l}{ }^{1} \text { Instituto Tecnológico de Chiná, Calle } 11 \\
\text { S/N entre } 22 \text { y } 28 \text { Chiná, San Francisco de } \\
\text { Campeche, Campeche. CP } 24520 .\end{array}$ \\
\hline $\begin{array}{l}{ }^{2} \text { Universidad Pública de El Alto, Área de } \\
\text { Ciencias Agrícolas, Pecuarias y Recursos } \\
\text { Naturales, Kallutaka Km } 35 \text { Carretera La } \\
\text { Paz - Desaguadero. La Paz, Bolivia. }\end{array}$ \\
\hline *Dirección de contacto: \\
\hline $\begin{array}{l}\text { Instituto Tecnológico de Chiná, Calle } 11 \\
\text { S/N entre } 22 \text { y } 28 \text { Chiná, San Francisco de } \\
\text { Campeche, Campeche. CP } 24520 .\end{array}$ \\
\hline $\begin{array}{l}\text { Martínez-Puc Jesús Froylán } \\
\text { E-mail address: } \\
\text { apisfroymap@yahoo.com.mx }\end{array}$ \\
\hline Palabras clave: \\
\hline $\begin{array}{l}\text { Apoidea, } \\
\text { polinización, } \\
\text { meliponinos, } \\
\text { Apis mellifera. }\end{array}$ \\
\hline $\begin{array}{l}\text { J Selva Andina Anim Sci. } \\
2014 ; 1(2): 28-34 .\end{array}$ \\
\hline Historial del artículo \\
\hline $\begin{array}{l}\text { Recibido junio, } 2014 . \\
\text { Devuelto noviembre } 2014 \\
\text { Aceptado diciembre, } 2014 . \\
\text { Disponible en línea, diciembre } 2014 .\end{array}$ \\
\hline $\begin{array}{l}\text { Editado por: } \\
\text { Selva Andina Re- } \\
\text { search Society }\end{array}$ \\
\hline Key words: \\
\hline $\begin{array}{l}\text { Apoidea, } \\
\text { pollination, } \\
\text { stingless bees, } \\
\text { Apis mellifera. }\end{array}$ \\
\hline
\end{tabular}

\section{Resumen}

El presente trabajo de investigación tiene el objeto de revisar información científica en relación a la taxonomía su diversidad, comportamiento y características de grupo, importancia de las abejas y las amenazas que enfrentan en la actualidad.

Los reportes señalan la existencia de 20000 a 40000 especies, incluidas en 443 géneros y siete familias, acorde a su forma de vida, las abejas se clasifican en abejas solitarias, abejas sociales las cuales a su vez se dividen en eusociales y parasociales, finalmente se encuentran las abejas cleptoparásitas. Las abejas juegan un papel importante en la polinización de los cultivos, sin embargo en la actualidad se enfrentan a una serie de problemas entre las que sobre sale la deforestación, el cambio de uso de suelo, la contaminación. Con el presente trabajo se llega a la conclusión de que existe una diversidad de especies de abejas (Hymenoptera: Apoidea), es muy importante en la polinización, sin embargo enfrenta más de un problema en la actualidad en Yucatán, México.

(C) 2014. Journal of the Selva Andina Animal Science. Bolivia. Todos los derechos reservados.

\section{Abstract}

This research has the purpose of reviewing scientific information in relation to taxonomy diversity, behavior and group characteristics, importance of bees and the threats facing today. Reports indicate the existence of 20000-40000 species, including 443 genera and seven families, according to their lifestyle, bees are classified as solitary bees, social bees which in turn are divided into eusocial and shareholders, finally kleptoparasites are the bees. Bees play an important role in the pollination of crops, however today face a number of problems including deforestation goes on, changing land use, pollution. With the present paper concludes that there is a diversity of bees (Hymenoptera: Apoidea), is very important in pollination, however faces more of a problem today in Yucatan, Mexico. 


\section{Introducción}

Actualmente un problema para la sociedad es la pérdida de la biodiversidad de especies como las abejas por efecto de la deforestación de los bosques, contaminación medio ambiental, monocultivos y otros, estos han generado una mayor atención al estudio de la diversidad biológica, y los efectos de las actividades humanas. Dentro de la biodiversidad en México, un grupo importante de estudio son las abejas, ya que en este grupo se encuentran los insectos que podrían ser los más benéficos y de mayor importancia económica directa para el hombre (LaSalle \& Gauld 1993). Varias especies de plantas requieren del servicio de polinización realizada por las abejas para producir sus frutos, que se considera de vital importancia.

Esto ha generado el interés por conocer la fauna de abejas de México que no es reciente y en un periodo mayor a 230 años, Cockerell, Timberlake, Cresson, Smith, LaBerge y Michener han contribuido con el 69.2\% de las especies conocidas (Ayala et al. 1996). Por lo tanto el presente trabajo de investigación pretende revisar información científica en relación a la taxonomía su diversidad, comportamiento y características de grupo, impor-tancia de las abejas y las amenazas que enfrenta en la actualidad, información que es relevante para comprender la necesidad de indagar más sobre la diversidad de estos himenópteros en México.

\section{Taxonomía y diversidad de especies de abejas}

Actualmente se estima que la diversidad de abejas silvestres a nivel mundial es de aproximadamente 20000 especies, incluidas en 443 géneros y siete familias, cinco de lengua corta (Stenotritidae, Colle- tidae, Andrenidae, Halictidae, Mellitidae) y dos de lengua larga Megachilidae y Apidae; (Michener 2007). Sin embargo, también se estima que el número podría llegar hasta 40000 especies (Roubik 1989). El Neotrópico es rico en apifauna y se reconocen alrededor de 5000 especies (Freitas et al. 2009), en México se encuentran cerca de 2000 especies (Ayala et al. 1993, 1996). Es importante resaltar que la región madrense árida y semiárida a lo largo del límite entre los Estados Unidos y México aloja la mayor cantidad de apifauna del mundo (Ayala, 1998). La subfamilia apinae está constituida por cuatro tribus Bombinini (abejorros), Apini (abejas melíferas), Euglossini (abejas de las orquídeas) y meliponini, abejas sin aguijon, (Miche-ner 2007).

El género Bombus Latreille comprende aproximadamente unas 250 especies (Michener 2007). En la región Neotropical y región Andina se tienen al menos 42 especies registradas en una variedad de hábitats. En México se tienen registros de al menos 14 especies (Abrahamovich et al. 2004).

La tribu Euglossini está formada por cinco géneros, Aglae, Eufriesea, Euglossa, Eulaema y Exaeretes (Michener 2007). En Yucatán se reportan cuatro especies: Eufriessea mexicana, Euglossa viridissima, Eulaema polychroma y Exaerete smaradigma (Reyes 2009).

En relación a la tribu Meliponini en el Neotrópico se registran 33 géneros y 391 especies (Freitas et al. 2009). En México se tienen identificado un total de 11 géneros y 46 especies (Ayala 1999), y en Yucatán se registran al menos 16 especies (QuezadaEuán 2005). Especies a las que se debe dar condiciones ambientales para su existencia. 


\section{Comportamiento}

De acuerdo a sus formas de vida, las abejas se clasifican en abejas solitarias, abejas sociales las cuales a su vez se dividen en eusociales y parasociales, finalmente se encuentran las abejas cleptoparásitas (Michener 2007).

Las abejas solitarias son aquellas que construyen sus nidos y aprovisionan las celdas con alimento para cada una de sus crías, sin la cooperación de otras hembras (Michener 20007). Las abejas cleptoparásitas son aquellas en donde las hembras depositan sus huevecillos en los nidos de otras especies de abejas y su progenie consume el alimento asignado para las larvas anfitrionas (Michener 2007).

En México la mayoría de las especies $70 \%$ de los géneros y $90 \%$ de de las especies son recolectoras de polen y solitarias o semisociales; $17 \%$ y $7 \%$ respectivamente son cleptoparasitas y el $13 \%$ y el $3 \%$ son altamente sociales (Ayala et al. 1998). En Yucatán se ha encontrado que el $34 \%$ de las especies tienen un comportamiento solitario, el 9\% son cleptoparásitas, el 50\% son parasociales y únicamente el $7 \%$ puede ser considerado como eusociales (Novelo-Rincón et al. 2003).

\section{Características de cada grupo}

Las abejas de la tribu Bombini están incluida en un solo género Bombus son abejas de tamaño medio (9 $\mathrm{mm}$ ) a muy grande $(22 \mathrm{~mm})$. Las garras de las hembras presentan una hendidura, el arolium es pequeño pero se encuentra presente, las alas presentan una nervadura bastante fuerte.

Las abejas de la tribu Euglosinini desempeñan un papel muy importante en los sistemas de polinización neotropicales. Los machos polinizan cientos de especies de orquídeas en la región neotropical mientras que las hembras rara vez lo hacen. Las abejas de las orquídeas (Eulaema, Eufresea, Euglossa y sus cleptoparásitas Exaerete y Aglae), se restringen al neotrópico, pese a la gran disparidad en extensión continental de los países de Centroamerica y México sus números decrecen con el incremento en latitud (Ayala et al. 1998).

Uno de los grupos taxonómicos mejor estudiados y los cuales pueden ser considerados como buenos polinizadores en cultivos de origen Neotropical, son las especies pertenecientes a la tribu meliponini (Quezada-Euán 2009). Actúan en el mantenimiento de la biodiversidad y en la conservación de la flora nativa, así como en la polinización de plantas cultivadas ya sea en invernaderos o al aire libre (NatesParra 2006). Las abejas sin aguijón o meliponinos se pueden distinguir del resto de los Apidae porque presentan una gran reducción de la venación de las alas anteriores, la falta de aguijón, uñas simples y presentar una línea de pelos gruesos en la margen distal de las tibias posteriores (Quezada-Euán 2005).

\section{Importancia de la abeja melífera en la poliniza- ción de cultivos}

La polinización juega un papel muy importante en la producción de los cultivos agrícolas y de las plantas silvestres (Taha \& Bayoumi 2009). Se calcula que más del $80 \%$ de las 250 mil plantas con flor conocidas en el mundo requieren de polinización para llevar a cabo su reproducción sexual (Kears et al. 1998, Coro-Arizmendi 2009). Sin embargo, los polinizadores de plantas silvestres y de cultivos agrícolas incluyen una amplia variedad de organismos, no únicamente abejas, y no solo abejas del género Apis (Roubik 1995).

Se estima que el $30 \%$ de los alimentos consumidos por los seres humanos derivan de plantas poliniza- 
das por abejas, actualmente existe una dependencia de los servicios de polinización hacia la especie $A$. mellifera en los cultivos agrícolas (Slaa et al. 2006). Una muestra de ello son los servicios prestados por concepto de polinización que suma unos 117000 millones de dólares anuales a nivel mundial. Es importante mencionar que al menos el $84 \%$ de los cultivos producidos en la Unión Europea dependen de la polinización de la abeja melífera y abejorros (Kearns et al. 1998).

En los Estados Unidos cuatro millones de colmenas producen miel y cera con un valor superior a los 100 millones de dólares. Sin embargo, al prestar el servicio de polinización a los cultivos se obtiene 10 veces ese valor en la producción (McGregor 1976, Guzmán, 2000). No obstante, en la actualidad en el Continente Europeo y en los Estados Unidos de Norteamérica, se ha presentado una elevada mortalidad de abejas de la especie $A$. mellifera (Higues et al. 2006, Higues et al. 2008a), así como una reducción en la población de abejorros (Cameron et al. 2009), lo cual ha ocasionado preocupación debido a la falta de servicios de polinización (Slaa et al. 2006).

\section{Importancia de los polinizadores nativos}

La polinización de cultivos utilizando a las abejas nativas sin aguijón es una actividad potencial que aún se encuentra poco difundida. Se puede considerar a las abejas sin aguijón como buenos polinizadores de cultivos de origen Neotropical como tomates, chiles, pimientos, aguacates y cucurbitáceas (Quezada-Euán 2009). En Yucatán se han realizado diversos estudios sobre la polinización de plantas locales con meliponinos, entre los que destacan el aguacate (Persea americana), donde se identificaron cuatro especies de abejas visitantes entre ellas tres meliponinos (Nanotrigona perilampoides, 31
Scaptotrigona pectoralis y Trigona nigra) y $A$. mellifera (Can-Alonso et al. 2005).

Figura 1 Pérdida de vegetación a causa de incendios

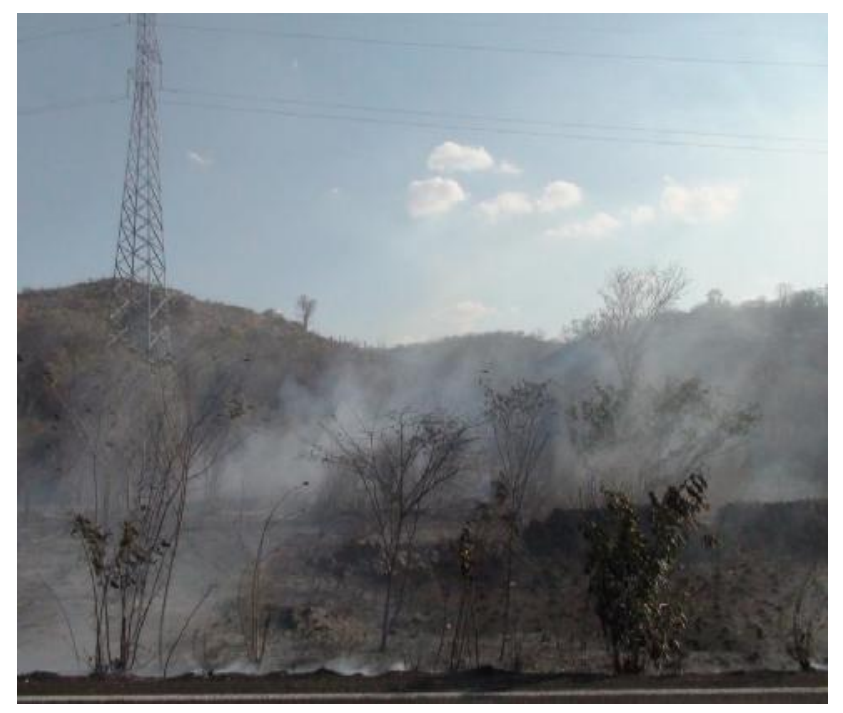

Figura 2 Contaminación ambiental debido al manejo inadecuado de la basura

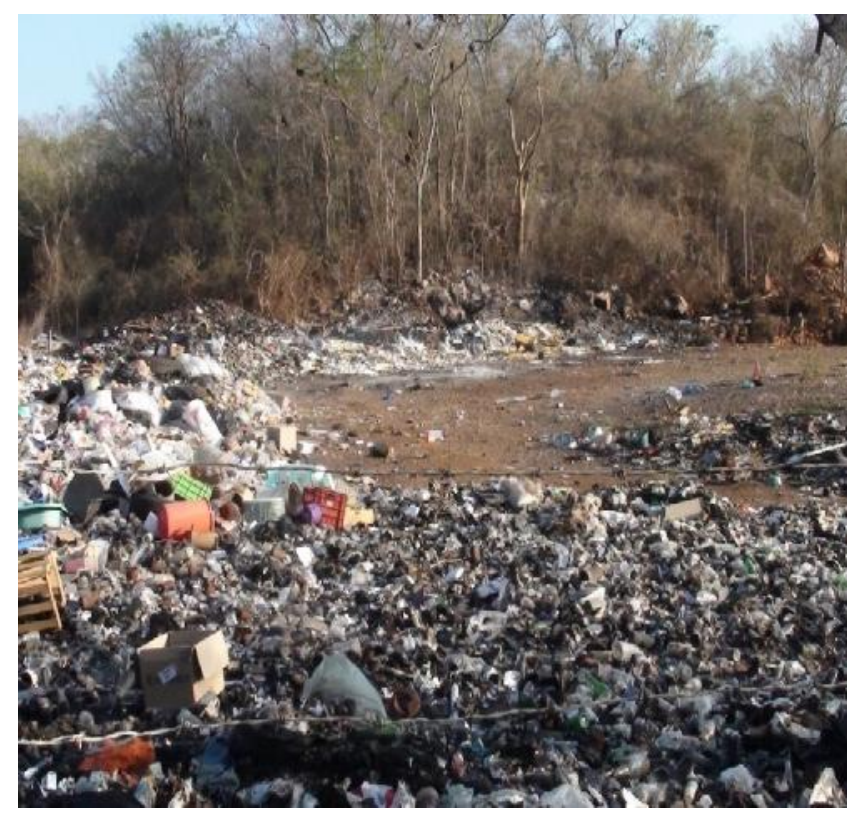

Se ha demostrado la eficiencia de las abejas en cultivos de Solanaceas, entre los que se encuentra el tomate (Lycopersicon esculetun Miil.) cultivado a 
cielo abierto (Macías 2001, Palma et al. 2008, Macías-Macías et al. 20013, observándose un incremento en el número de semillas y en el amarre de los frutos (Macías-Macías, 2001), y el chile habanero (Capsicum chinense Jacq.) en condiciones de invernadero (Cauich 2004, Palma et al. 2008), así como también en el achiote (Caro 2010).

Figura 3 Establecimiento de monocultivos

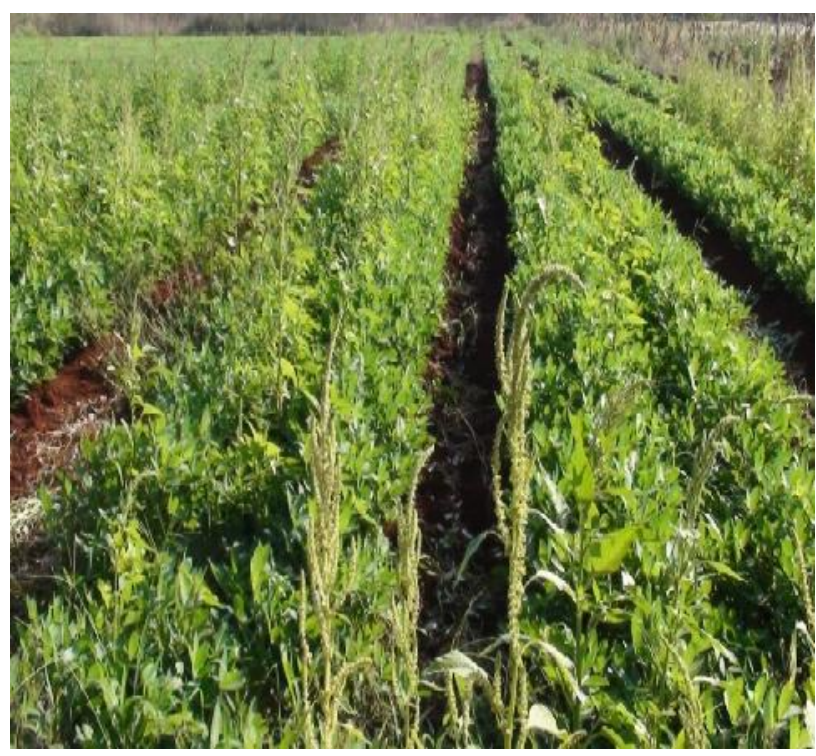

\section{Amenazas para los polinizadores}

La deforestación y la intensificación de los cultivos agrícolas representan una amenaza para la apifauna (Freitas et al. 2009). La transformación del medio natural para pasar a la explotación agrícola del suelo ha ocasionado un efecto muy negativo sobre la apifauna: por un lado, se han destruido los lugares más propicios para el anidamiento, y por otro, se han eliminado las especies vegetales que aportaban la fuente de alimento original.

La deforestación es un fenómeno que se ha manifestado gradualmente y se ha agudizado en los últimos años, trayendo como consecuencia una reducción notable en la superficie de las selvas de México y del mundo (Sánchez \& Rebollar 1999). En México se estima la pérdida de 347600 ha al año (Freitas et al. 2009). En Yucatán se calcula que más del $24 \%$ de la superficie se encuentra fragmentada, lo cual tiene un efecto negativo en la biodiversidad.

\section{Conclusión}

De acuerdo a información revisada existen aproximadamente de 20000 a 40000 especies, incluidas en 443 géneros y siete familias, de acuerdo a sus formas de vida, las abejas se clasifican en abejas solitarias, abejas sociales las cuales a su vez se dividen en eusociales y parasociales, finalmente se encuentran las abejas cleptoparásitas. La importancia de estas especies radica en la polinización que juega un papel muy importante en la producción de los cultivos agrícolas y de las plantas silvestres.

Sin embargo, la deforestación, la intensificación de los cultivos agrícolas, la contaminación (figuras 1, 2 y 3) representan una amenaza para la existencia de la apifauna, la que debe ser considerada por el ser humano, eliminar sería el peor error que atentaría a la seguridad y soberanía alimentaria.

\section{Conflicto de interes}

El presente trabajo no genera conflictos de interes.

\section{Literatura citada}

Abrahamovich HA, Diaz N, Morrone J. Distributional patterns of the Neotropical and Andean species of genus Bombus (Hymenoptera: Apidae). Acta Zool Mex. 2004; 20(1): 99-117. 
Ayala R, Griswold T, Bullock SH. The native bees of Mexico. En: Ramamoorthy, TP, Bay R., Lot A, Fa, J. (Eds.). Biological diversity of Mexico, Origin and distribution. Oxford. University press. 1993; 179-227 pp.

Ayala R, Griswold T, Yanega D. Apoidea (Hymenoptera). In: Llorente-Bousquets J. García A, \& González E (Eds.). Biodiversidad, Taxonomía y Biogeografía de artrópodos de México: Hacia una síntesis de su conocimiento. UNAM, CONABIO. Mexico, D.F. 1996; 27: 423-464 pp.

Ayala R, Griswold TL, Bullock SH. Las Abejas Nativas de México. In: Ramamoorthy, T.P., Bay, R., Lot, A. Fa, J. (eds.), Diversidad biológica de México, Orígenes y Distribución. Instituto de Biología, UNAM, México. 1998; 179-225 pp.

Ayala R. Revisión de las abejas sin aguijón en México (Himenoptera: Apidae: Meliponni). Folia Entomol Mex. 1999; 106-123.

Cameron S, Lozier S, Strange J, Koch S, Cordes N, Solter L, et al. Patterns of widespread decline in North American bumble bees. PNAS 2011; 108(2):662-667.

Can-Alonzo C, Quezada-Euan JJG, Xiu-Ancona P, Moo-Valle H, Valdovinos-Nunez GR, MedinaPeralta S. Pollination of "criollo" avocados (Persea americana) and the behaviour of associated bees in subtropical Mexico. J Apic Res. 2005; 44:3-8.

Caro VAM. Contribución de tres especies de abejas sociales (Hymenoptera: Apidae) en la eficiencia de la polinización del achiote (Bixa orellana L.). Tesis Maestría. Opcion: Apicultura Tropical. 51 p. 2010.

Cauich-Muños O, Quezada-Euán JJ, Macias-Macias JO, Reyes-Oregel V, Medina-Peralta S, ParraTabla V. behaviour and pollination efficiency of Nannotrigona perilampoides (Hymenoptera: 33
Meliponini) on greenhouse tomatoes (Lycopersicon esculentum) in subtropical Mexico. J Econ Entomol. 2004; 97:475-481.

Cauich-Muños O. Eficiencia de la abeja nativa Nannotrigona perilampoides $\mathrm{Cr}$. (Apidae: Meliponini) en la polinización del chile habanero (Capsicum chinensi Jacq.) en cultivo bajo cobertura en Yucatán, México.2004.

Coro-Arizmendi. La crisis de los polinizadores. CONABIO. Biodiversitas. 2009; 85; 1-5.

Freitas S, Penny ND, Adams PA. A revision of the New World genus Ceraeochrysa (Neuroptera: Chrysopidae). Proc Calif Acad Sci. 2009; 60:503-610.

Guzmán M. Efecto de las visitas florales por insectos en la producción de frutos de rambutan (Nephelium Lappaceum L.), en el Soconusco, Chiapas, México. Tesis Maestría. UNACH. 2000; $1-5$ pp.

Higues M, Martin R, Meana A. Nosema ceranae, a new microsporidian parasite in honeybees in $\mathrm{Eu}-$ rope. J Inverteb Pathol. 2006; 92(2):93-95.

Higues M, Martín-Hernández R, Botías C, Bailón E G, González-Porto AV, Barrios L, del Nozal M J, Bernal J L, Jiménez JJ, Palencia PG, Meana A. How natural infection by Nosema ceranae causes honeybee colony collapse. Environmental Microbiology, 2008a; (10):2659-2669.

Kearns CA, Inouye DW, Waser N. Endangered mutualism: The conservation of plant-pollinator interactions. Annu Rev Ecol Syst. 1998; 29:83106.

LaSalle J, Gould ID. Hymenoptera: their diversity and their impact on the diversity of other organisms. In LaSalle J. y Gauld ID (eds.) Hymenoptera and biodiversity. CAB International. UK. 1993; 257-277 pp. 
Macias MJO, Quezada-Euán JJG, Parra-Tabla V, Reyes OV. Comportamiento y eficiencia de polinización de las abejas sin aguijón (Nannotrigona perilampoides) en el cultivo del tomate ( $L y$ copersicon esculentum) bajo condiciones de invernadero en Yucatán, México. II Seminario Mexicano sobre abejas sin aguijón. 2001; 119$124 \mathrm{pp}$.

Macías-Macías JO, Quezada - Euán JJG, Tapia GJM, Contreras - Escareño F. Pecoreo y desarrollo de nidos de melipona colimana (hymenoptera: meliponini) en clima templado de Jalisco, México. VIII Congreso Mesoamericano de abejas nativas: Biología, Cultura y Uso Sostenible. 2013; 30-37 pp.

McGregor SE. Insect pollination of cultivated crop plants. Washington, US Dept. Agric. 1976; 411 pp.

Michener CD. The Bees of the World. ( $2^{\text {nd }}$ Edition). Johns Hopkins University Press. 2007; 953 pp.

Nates-Parra G. Abejas Corbiculadas de Colombia: Hymenoptera: Apidae. Universidad Nacional de Colombia. Facultad de Ciencias. Bogotá DC. 2006; 156 pp.

Novelo-Rincón LF, Delfin-Gonzalez H, Ayala R, Contreras-Acosta HH. Community structure of native bees in four vegetation types in the dry tropics of Yucatan, Mexico. Folia Entomol Mex. 2003; 42:177-190.

Palma G, Quezada-Euán JJG, Reyes-Oregel V, Meléndez-Ramírez V, Moo-Valle H. Production of greenhouse tomatoes (Lycopersicon esculentum) using Nannotrigona perilampoides, Bombus impatiens and mechanical vibration (Hym.: Apoidea). J Appl Entomol. 2008; 132:79-85.
Quezada-Euán JJG. Biología y uso de las abejas sin aguijón de la Península de Yucatán, México (Hymenoptera: Meliponni). Universidad Autónoma de Yucatán. Mexico. 2005112 pp.

Quezada-Euán JJG. Potencial de abejas nativas en la polinización de cultivos. Act Biol Colomb. 2009; 14(2): 169-172.

Reyes NEA, Meléndez RV, Delfin GH, Ayala R. Abejas silvestres (Hymenoptera: Apoidea) como bioindicadores en el Neotrópico. Trop Subtrop Agroecosyst. 2009; 1-13.

Roubik DW. (eds). Pollination of cultivated plants in the tropics. FAO, Food and Agriculture Service Bulletin Number 118, Rome, Italy 1995.

Roubik DW. Ecology and Natural History of Tropical Bees. Cambridge Tropical Biology Series, Cambridge University Press. Cambridge, UK. 1989.

Sánchez ALR, Rebollar DS. 1999. Deforestación en la Península de Yucatán, los retos que enfrentar. Madera y Bosques. 1999; (5):3-17.

Slaa EJ, Sanchez-Chavez LA, Malagodi-Braga KS, Hofstede FE. Stingless bees in applied pollination: practice and perspectives. Apidologie 2006; 37; 293-315.

Taha EKA, Bayoumi YA. The value of honey bees (Apis mellifera $\mathrm{L}$.) as pollinators of summer seed watermel on (Ci trul l us lanatus colothynthoides L.) in Egypt. Acta Biol Szeged. 2009; 53(1):3337. 